\title{
"Les élèves face aux devoirs et aux copies corrigées : bavardage inutile, réactions aberrantes ou discours révélateur ?"
}

\section{Bernard Delforce}

\section{(2) OpenEdition}

\section{Journals}

Édition électronique

URL : http://journals.openedition.org/edc/3250

DOI : 10.4000/edc.3250

ISSN : 2101-0366

\section{Éditeur}

Université de Lille

Édition imprimée

Date de publication : 1 février 1986

Pagination : $91-116$

ISSN : 1270-6841

Référence électronique

Bernard Delforce, " "Les élèves face aux devoirs et aux copies corrigées : bavardage inutile, réactions aberrantes ou discours révélateur ?" ", Études de communication [En ligne], $7 \mid$ 1986, mis en ligne le 01 avril 2012, consulté le 10 décembre 2020. URL : http://journals.openedition.org/edc/3250 ; DOI : https://doi.org/10.4000/edc.3250

Ce document a été généré automatiquement le 10 décembre 2020.

(c) Tous droits réservés 


\title{
"Les élèves face aux devoirs et aux copies corrigées : bavardage inutile, réactions aberrantes ou discours révélateur ?"
}

\author{
Bernard Delforce
}

1 De la même manière qu'on peut mettre en évidence chez les enseignants un discours typé relatif à l'évaluation et à la correction des copies, on peut s'efforcer de recueillir, d'observer et d'analyser le discours que les devoirs et le retour des copies produisent chez les élèves.

2 Soit, dira-t-on. Ce serait, en effet, accorder un intérêt égal aux deux catégories d'acteurs (enseignants/élèves) que met en scène la situationde correction des copies. Mais, les propos que tiennent les élèves à ce sujet constituent-ils vraiment undiscours ?Quel intérêt, autre qu'anecdotique évidemment, peut présenter le recueil de ce "bavardage", de ce "bruit de fond" de la classe, parasite et dépourvu d'une quelconque originalité ?

3 C'est à ces "réserves" qu'on voudrait d'abord s'efforcer de répondre.

I - Le discours des élèves : un bavardage sans intérêt?

1.1) Un discours spontané et homogène.

4 Donner un devoir et, surtout, le rendre corrigé,voilà qui provoque systématiquement, on l'a tous constaté, voire déploré, la parole chez les élèves: questions, remarques, récriminations diverses, bavardages et échanges avec les voisins... Cette parole peut prendre des formes diverses : elle peut êtreadressée explicitement au professeur ou aux voisins immédiats. Elle peut aussi prendre la forme de propos lancés à la cantonade, adressés à personne et destinés à tous, ou encore d'à parte, destinés à certains mais audibles aussi pour d'autres. Cette parole. s'exprime encore sous des formes non verbales: réactions corporelles, gestes, mimiques... où ce qui se vit et se pense intérieurement se donne à lire au regard des autres. Cette situation produit donc bien "du" discours chez les élèves. 
5 Mais ce discours est aussi relativement clos,homogèneet peu diversifié : il fonctionne, comme on le verra plus loin, à partir d'un nombre limité de thèmes ou de topoï et met en œuvre des modalités de raisonnement typées. D'ailleurs, les enseignants eux-mêmes ne s'y trompent pas qui disent avoir l'impression d'entendre toujours un peu la même chose de classes en classes, de devoirs en devoirs, une année après l'autre : "c'est toujours un peu pareil".

1.2) Un discours facilement accessible.

6 Les éléments de ce discours sont, en outre, facilementaccessiblespour l'enseignant. D'abord, parce qu'ils s'offrent en classe à l'écoute et au regard de l'enseignant bien qu'il ne l'ait ni souhaité ni prévu: il ne peut y échapper. Il est même, par l'organisation traditionnelle de l'espace scolaire, le mieux placé pour le voir comme pour l'entendre. Curieusement d'ailleurs, même les propos qui ne lui sont pas directement et explicitement adressés (a parte, propos à la cantonade, gestes et mimiques...) paraissent souvent énoncés de façon telle qu'il puisseaussien être, s'il le veut, le récepteur. Mais à la condition, toutefois, d'assumer alors d'être, lui, à l'initiative de la communication... que l'élève vient pourtant d'enclencher. Il s'agit donc toujours, peu ou prou, d'un discoursqui lui est destiné, espèce de discours "interdit" qui chercherait malgré tout à se faire entendre.

7 Mais il existe, en outre, d'autres occasions pour l'enseignant d'un contact avec ce discours des élèves sur les devoirs et la correction des copies. D'abord, les enseignants ont euxmêmes d'abord été élèves et gardent des souvenirs de cette situation: comment la vivaient-ils alors? qu'en disaient-ils? Qu'en disaient aussi leurs camarades? Il arrive aussi que l'enseignant se trouve mis en présence de ce discours hors de l'école: comme parent, comme ami, comme voisin, voire comme usager ordinaire des transports en commun, il entend des apprenants, dont il n'est pas le professeur, parler de leurs difficultés devant un devoir, raconter "honnêtement" comment ils s'y sont pris réellement, se plaindre des corrections des collègues ou, au contraire, les louer... Enfin, parce qu'il a lui-même eu à faire des devoirs, parce qu'il élabore encore, éventuellement, des corrigés, parce qu'il aide, peut-être, aussi des apprenants qui ne sont pas ses élèves... l'enseignant détient, au moins potentiellement, toutes sortes d'informations sur l'activité de production des devoirs, sur les problèmes qu'elle soulève, les difficultés, questions, hésitations dont elle est le lieu, les moyens d'y répondre... et les réactions que suscite ensuite la correction.

8 Les enseignants ont donc de multiples occasions d'être, de fait, mis en présence de ce discours des élèves. Or, curieusement, par une sorte de phénomène de "méconnaissance", ce discours leur est, à la fois, parfaitement familier et connu et, en fin de compte, relativement étranger. Tout se passe, en définitive, comme si les enseignantssavaient sans vouloir savoir.L'explication de ce phénomène pourrait bien se trouver dans lesattitudes habituelles des enseignants à l'égard de ce discours.

1.3) Un discours sans intérêt et faux ?

9 Quand on les interroge à ce sujet, les enseignants disent ne pas faire grand-chose de ce discours et ne s'être jamais vraiment préoccupés de le recueilliret de l'enregistrer de façon systématique dans la mesure où il leur sembledépourvu d'un quelconqueintérêt: quelles connaissances utiles peuvent donc apporter à l'enseignant des réactions aussi "tranchées", "décousues", "à l'emporte-pièce"... ? N’est-cepas, en outre, verser dans un "sentimentalisme" mal venu que de s'intéresser aux difficultés que les élèves prétendent 
avoir rencontrées dans l'élaboration de leur devoir? Au mieux, on est "attentif" à ce discours comme à undiscours parasitequi fait irruption sans qu'on l'ait prévu et souhaité et qui porte sur des éléments de la correction qui divergent fortement d'avec les attentes du professeur. Un discours, donc, qu'il importe avant tout de "contenir" et auquel il faut savoir rapidement mettre fin. Il est donc exclu de contribuer à ce qu'il s'exprime plus complètement et plus systématiquement. En définitive, c'est encore à son caractère mensonger et fauxqu'on se montre le plus sensible. Ainsi, on avouera, par exemple, qu'on a déjà tenté, quelquefoisen classe, mais surtout hors de la situation scolaire, avec des apprenants dont on n'était pas le professeur, de convaincre que les enseignants étaient sans doute plus "consciencieux" ou moins "sadiques" que les élèves le prétendaient. Par ailleurs, si onaccepte parfois de dire qu'on ressent ce discours comme "menaçant" à partir de la charge critique qu'il contient, on a tôt fait de la présenter comme uniquement défensif :undiscours de parade des mauvais élèves.

1.4) Un discours précieux pour changer lespratiques?

10 Pourtant, le recueil systématique et l'analyse de ce discours ne nous semblent pas pouvoir être exclus d'une réflexion sur la correction des copies et d'une tentative d'en modifier lespratiquesetles effets.

Plutôt que de tenir ce discours àdistance, voire de l'annuler, en privilégiant d'emblée les hypothèses les plus invalidantes pour le sujet qui le tient, nous nous efforcerons d'entendre ce discours "pour de bon", en essayant de tirer parti de ce qu'il nous apprend sur ce qui se joue et se vit réellement en classe autour des devoirs et de la correction des copies.

D'abord, ce discours se présente, en fait, commel'un deseffets des pratiques actuelles :il est, en définitive, uneréactionà ces pratiques dont on peut chercher à comprendredans quelle mesure celles-ci l'engendrent, lui laissent place ou le rendent nécessaire.

Il se pourrait bien aussi que ce discours soit partiellement àl'origine des pratiques actuelles : on peut penser, en effet, que si l'enseignant s'efforce de concevoirdes pratiqueslégitimes pour l'institution, il ne se désintéresse jamais totalement de les rendrerecevablespour les élèves. Modifier l'écoute et la compréhension de ce discours par les enseignants peut donc, en retour, contribuer à modifier leurs pratiques.

Effet et origine des dispositifs pédagogiques, ce discours pourrait bien n'être, aussi, que le reflet, en même temps qu'il la susciterait et l'entretiendrait, d'unerelation faussée entre l'enseignant et l'apprenantet qui jouerait, alors, commeverroude la situation scolaire :un peu à la manière de ces couples toujours en conflit et qui se disputent à perte de vue sans jamais se séparer, chacuntrouverait, ici, dans ce discours, à se conforter dans sa place, tout en s'en plaignant, et à tirer parti des "bénéfices secondaires" qu'il peut en escompter plutôt que d'affronter le changement et ses exigences.

Si donc l'analyse de ce discours peut nous permettre de mieux comprendre ce qui se joue dans la situation pédagogiqueindépendamment des intentions etdes projets conscients des acteurs,il pourrait aussi nous aider àcomprendre ce qui, chezl'élève, modèle et donne sens à ses attitudes et à ses réactions,ce qui organise la forme particulière que prennent ses activités.Or, on ne peut véritablement accéder à cette compréhension que si l'on accepte d'analyser aussi ce discoursdu point de vuede celui qui l'émet -et pour qui le discours est toujours cohérent et pertinent sur la base des représentations dont il est porteur - et non du point de vue de l'autre dans l'interaction (ici, l'enseignant), pour qui le discours d'en face ne peut bientôt plus être perçu que comme obstacle - volontaire? - à son projet, bizarrerie 
incompréhensible, discours faux et rusé, attitude injustifiée, quand ce n'est pas inqualifiable.

16 C'est dans les perspectives qu'on vient d'évoquer qu'on a proposé, en stage, aux enseignants d'essayer dereconstituer,ensemble, le plus complètement possible,le discours des élèvessur les devoirs et la correction des copies. Il s'agissait tout à la fois pour nous de montrer aux enseignants qu'ils détenaient une information, souvent partielle, sur ce discours et que cette information constituait un appui précieux pour le changement. On souhaitait ainsi favoriser ultérieurement uneattention plus fine,un recueil plus systématique de ce discours et fournir pour soninterprétationunediversité d'hypothèses plausibles(plutôt qu'une explication unique et expérimentalement démontrée**

II - Reconstitution du discours (verbal/non verbal) des élèves

2.1) Les élèves devant la copie corrigée.

17 - Observation 1 : l'attente exclusive de la note.

De l'ensemble des éléments de la copie que reçoit l'élève (son texte initial auquel sont venus s'ajouter des annotations en marge, une appréciation générale en têtede la copie et une note), les enseignants observent - et s'en plaignent - que c'est très largement surla noteque se concentre son intérêt. C'est elle qu'on attend, c'est d'elle qu'on prend prioritairement et presque exclusivement connaissance. Quand lereste est lu, c'est dans un ordre précis : l'appréciation générale, puis l'ensemble des annotations. L'élève relit rarement l'intégralité de son texte initial.

18 - Observation 2 : une intense activité d'échange.

A ce moment de prise de connaissance des copies succède alors, ouvertement ou de façon plus discrète, une intense activité d'échange entre les élèves : on s'échange les copies ou, au minimum, on se renseigne sur les notes obtenues par d'autres. On compare les notes, les appréciations, on confronte, oncommente. Deux préoccupations paraissent soustendre cet échange: certains semblent comparer les notes dans une perspective de compétitionpour établir une espèce de palmarès dans lequel se situer. D'autres sont plutôt préoccupés de vérifier que la correction a respecté un critère dejustice et d'équitéentre tous.

19 - Observation 3 : des réactions théâtralisées.

La remise des copies provoque aussi desréactionsqu'on peut d'abord lirephysiquement.

20 Que la note soit bonne ou mauvaise, la réaction la plus fréquente estl'étonnementqu'on théâtralise sous la forme de lasurprise heureuseou, au contraire, sous celle de ladéception.

21 On note aussi des réactions qu'on désigne du terme degêneet qui proviennent aussi bien de l'élève qui a obtenu une bonne note (habituelle ou exceptionnelle pour lui) que de celui qui a obtenu une mauvaise note (tout à fait inhabituelle ou régulière).

22 Lesmauvaises notessemblent, quant à elles, provoquer des réactions spécifiques et plus théâtralisées encore. Certains jouentostensiblement l'absence d'effet :on théâtralise ainsi son silenceen "faisant la tête", on joue ledésintérêten lisant à peine sa copie et en la plaçant visiblement hors de portée du regard, on affiche sonindifférence. Al'inverse, on peut observer, chez d'autres, la manifestation d'une espèced'excès d'effetqui peut prendre la forme de lacolèrecontenue ou del'abattementallant parfois jusqu'aux larmes, voire celle de labravade("c'est moi qui ai eu la plus mauvaise note").

23 - Observation 4 : un discoursverbal récurrent.

Accompagnant l'échange des copies en venant appuyer ces réactions, surtout lorsque la 
note est mauvaise, on relève aussi toute une série dephrases typéesque les enseignants reconnaissent tous avoir déjà entendues.

Une première série deréactions témoigne d'uneattitudefataliste et résignéeface au verdict: "j'ai toujours la même note", "j'aurai jamais la moyenne", "j'ai toujours été nul en français"...

Les autres réactions, au contraire,s'opposentau verdict et lecontestent.Certaines accusent le professeur d'avoir gravementfaussé les règles du jeu: "c'est à la tête du client", "Décidément, il m'en veut", "Il y a des fayots" "L’an dernier, j'avais de bonnes notes"... Les plus nombreuses contestent lajusticedes critères utiliséspar l'enseignant et manifestent ainsi une incompréhension à l'égard du système d'évaluation. Elles pourraient toutes commencer par "c'est pas juste" : "On a écrit la même chose, on n'a pas la même note", "Pourtant j'en avais mis plus que lui", "tout ça j'enai parlé, je ne comprends pas pourquoi j'ai une si mauvaise note"; ou encore: "Pourtant, qu'est-ce que je l'avais travaillé ce devoir!" "Je savais, mais j'avais pas compris que c'était ça que vous vouliez qu'on fasse". Toutes font apparaître, en définitive, lesdeux critèresles plus valorisés par les élèves : les connaissancesetl'effort.Enfin, certaines réactions remettent en question lesoinque le professeur aurait apporté à lalecture des copies ; "j'en ai parlé, mais vous ne l'avez pas vu", "Vous n'avez presque rien écrit sur ma copie (ou: sur la 2ème page...)", "Il n’a pas compris ce que je voulais dire".

Il est intéressant de compléter ce discours par les"excuses"qu'invoquent, en général, les élèves quand ils doivent justifier une mauvaise note auprès de leurs parents. On y retrouve globalementl'accusation de subjectivité("Il ne peut pas me sentir", "il n'est pas d'accord avec mes idées"...), l'incompréhension descritèresutilisés ("Je ne comprends pas ce qu'il me reproche, ce qu'il veut...", "j'avais pourtant travaillé", "j'en avais mis plus que Untel"...) et laremise en cause de la qualité dé lecture("Il n'a pas bien lu ma copie"...). Mais viennent aussi s'y ajouter des arguments qui témoignent desliensétroits que nouent les élèvesentre enseignement et évaluation.D'une part, on remet en cause la qualité de l'enseignement dispensé préalablement: "le prof, ne sait pas bien expliquer", "on ne comprend pas ce qu'il veut"... D'autre part, on l'accuse d'avoir enfreint une règle implicitede l'évaluation qui voudrait qu'elle ne porte que sur ce qui a été effectivement appris ("Il nous a interrogé sur quelque chose qu'on n'avait pas fait", "on a eu un exercice qu'on n'avait jamais vu"...) et qu'elle tienne compte des résultats d'ensemble dugroupeclasse ("Il note dur", "tout le monde a eu une mauvaise note", "personne n'a eu la moyenne", "Il a voulu nous saquer"...).

On peut, par ailleurs, confronter cette image de l'évaluateur à celle, inverse, du "bon enseignant" pour les élèves : celui qui écoute, dialogue, comprend, respecte...

- Observation 5 : contestation explicite et négociation.

A côté de cette contestation publique mais implicitement adressée à l'enseignant, on peut aussi observer, dans certains cas, une autre forme de contestationplus proche de la négociation : on va voir le professeur "à la fin de l'heure".

D'une façon générale, cette intervention porte exclusivement sur lanoteet se présente rarement comme une contestation radicale. Elle peut prendredeux formesfréquentes :

Elle prend souvent - mais les enseignants disent ne pas s'y tromper - la forme d'unesimple demande d'explications :on vient demander pourquoi on a obtenu cette note. Mais pourquoi est-ce souvent perçu par le professeur comme par l'élève comme une contestation voilée qu'on formule avec un luxe de prudence et de circonlocutions? 
31 Elle peut prendre aussi la forme d'une tentative denégociationpour que la note soit relevée. Deux catégories d'arguments sont alors fréquents :l'injustice("j'avais pourtant travaillé, fait comme vous aviez dit...", "j'en ai mis plus qu'Untel...") etl'excessivesévérité("Vous m'avez mis 9, pour un point !"...).

2.2) Les élèves face au devoir à faire.

32 - Observation 6 : la pauvreté des informations chez l'enseignant.

Élémentle plus frappant: c'est sur ce point que les enseignants produisent le moins d'informations. Sans doute doit-on y voir le signe que cette question échappe à leur champ d'intérêt.

Or, on peut penser que certaines erreurs observables dans les devoirs proviennent des procédures de travail utilisées par les élèves et qu'une attention plus marquée envers le travail concretdes élèves ne manquerait pas d'intérêt (voir, par exemple, B. Delforce, 1983). Par contre, cette absence d'informations précises sur les modalités de réalisation des devoirs n'empêche pas les enseignants d'utiliser globalement "l'insuffisance du travail des élèves" comme l'une des hypothèses privilégiées pour expliquer les mauvais résultats scolaires. Pourtant, certains devoirs au moins s'effectuent en classe, sous la "surveillance" de l'enseignant, et il y aurait là un terrain d'observation fiable et facilement accessible sur les procédures de travail concrètes qu'utilisent les élèves.

34 Certains sont cependant attentifs à l'attitude des élèvesface au devoir proposé . ils notent l'inquiétudequ'il suscite chez certains élèves, y compris chez ceux qui, pourtant, réussiront. D'autres sont plutôt sensibles àl'incompréhensionde ce qu'il faut faire dont témoignent, à la fois, les regards et les questions posées : "est-ce qu'on peut faire ?", "estce qu'on doit faire ?" (pour des remarques convergentes, B. Delforce, 1982 a et 1983).

2.3) Les élèves face à la copie à corriger.

35 - Observation 7 : Des élèves non motivés ou désemparés?

D'abord, les enseignants s'accordent pour reconnaitre - et regretter - que le moment de correction générale intéresse peu les élèves qui ne se sentent pas concernés par ce qui est dit pour tout le monde, ni même, plus grave encore, par ce qui est pourtant dit à leur intention précise.

La reprise de leur propre copie par les élèves ne semble pas plus satisfaisante : certains regrettentl'indifférencedes élèves qui ne s'investissent pas dans la tâche qu'on leur demande; d'autres notent que les élèves ont plutôt l'airdésemparésqu'indifférents : ils ne savent pas comment s'y prendre pour retravailler leur copie. Signes de cette difficulté : les passages que les élèves remanient complètement alors qu'on n'attendait d'eux que des modifications très localisées ou, inversement,les modifications de simples détails quand c'est le passage entier qu'il faudrait reprendre ${ }^{1}$. Signes aussi de cette incertitude des élèves, quand le travails'effectue en classe : leurs sollicitations fréquentes. Ils demandent de l'aide ou cherchent à vérifier auprès de l'enseignant "si ça peut aller comme ça".

De façon générale, enfin, tous constatent que ce moment de correction restedécevant malgré les formes diversifiées qu'on a tenté de lui donner : corrections individuelles ou en groupe, correction chez soi ou en classe, notation ou absence de notes... Si certaines procédures apportent quelques améliorations dans l'attitude des élèves, elles ne font qu'atténuer des dysfonctionnements qui, cependant, restent prégnants.

III - Hypothèses d'interprétation 
位ons d'abord qu'il n'entre pas dans notre projet d'analyser ici, de façon exhaustive, le discours des élèves. D'une part, le matériel dont nous disposons se présente aussi comme le discours des élèvestel que le reconstituent des enseignants.Mais surtout, il s'agit essentiellement pour nous de chercher àmodifierles attitudesdes enseignants à l'égard de ce discours. C'est pourquoi, il nous parait plusefficace de proposer unesérie d'hypothèses variées et plausiblesplutôt qu'une seule explication tout à fait validée. Cette démarche vise à atténuer les attitudes les plus fréquentes des enseignants envers ce discours (désintérêt, mise à distance, assignation d'une signification univoque et définitive) pour développer chez eux, au contraire, uneattitude d'exploration et de questionnement: si mieux observer permet de mieux comprendre, en retour, des possibilités d'interprétation élargies favorisent une observation plus fine.

3.1) Professeurs/Élèves : deux discours en écho.

Ce qui frappe d'abord ce sont lessimilitudesentre ce discours et celui que les enseignants tiennent sur la correction des copies. On a, en effet, l'impression qu'il s'agit d'un même discours, globalement négatif, fait d'insatisfactions, de plaintes et de reproches ${ }^{2}$.

3.1.1.) Deux discours adverses ou inverses?

Ainsi, on ne peut qu'être frappé par l'évocation de l'ennui et del'absence d'intérêtqui s'attachent, tant chez les enseignants que chez les élèves, aux différentes activités qui composent l'évaluation : production du devoir, correction des copies, correction du texte initial par l'élève...

Mêmesdoutesaussi, chez les uns et chez les autres, sur la nature précise de l'activité et sur les façons de l'entreprendre, mêmesinquiétudesquant aux résultats. D'un côté, des élèves qui semblent désemparés aussi bien devant le devoir à faire que devant leur texte initial à corriger; de l'autre, des enseignants qui se demandent si ce qu'ils font correspond bien à ce qu' il faut faire, souvent démunis devant l'inefficacité relative de leur travail et qui cherchent à ce qu' on leur dise comment on pourrait s'y prendre pour réussir/être efficace. Comme si à l'angoisse devant la feuille blanche succédait l'angoisse devant le paquet de copies à corriger.

Mêmesregretsaussi d'uneabsence de changement:au "c'est toujours pareil de copies en copies", sensible chez les professeurs, semblent répondre les "j’ai toujours la même note" ou les "j'ai toujours été nul" des élèves.

Réactions similaires encore face àl'échecet mêmerecoursà l'accusationou àl'autoculpabilisation:si les uns imputent progressivement l'échec aux seuls élèves, les autres privilégient des arguments qui rendent les professeurs responsables de celui-ci.

Mêmeabsencenotable, enfin, dans le discours des uns comme dans celui des autres, d'une représentation précise del'activité del'autre: siles professeurs savent, en définitive, peu de choses sur les procédures de production et les conditions de l'activité scripturale chez les élèves, les élèves, de leur côté, n'évoquent jamais la correction que comme une activité "évidente" dont les "erreurs" ne peuvent être que volontaires ("Il m'en veut") ou résulter d'un manque de "travail" ou d'attention du professeur ("Il ne m'a pas bien lu").

En définitive, il ne manque pas d'éléments qui nous invitent à percevoir ces deux discours comme des variationssur une même trame plutôt que comme deux discours qui s'affrontent, comme deux discoursinversesau moins autantqu'adverses ${ }^{3}$.

3.1.2) Deux discours : un même système.

Études de communication, 7 | 1986 

peut, au moins,rééquilibrer lapart des remarques positivesen mettant l'accent, par exemple, sur ce que la première version qu'on a sous les yeux contient de prometteur pour la suivante. Sinon, comment prendre intérêt à améliorer quelque chose dans lequel on a investi de soi (cf. la nature des sujets proposés), si on vous le renvoie comme ne valant pas grand-chose? montre à l'élève que ce qu'on perçoit de son propreprojet d'écriture(voir, ici, Y. Reuter) peut parfaitement se réaliser... à condition qu'il s'y prenne autrement (non directivité sur le contenu, directivité sur la forme, voir B. Delforce 1982a) Le"recadrage"des inévitables difficultés rencontréespar les élèves dans l'activité d'écriture en les pointant comme signes d'abord de leursagacité("vous avez bien perçu les lieux problématiques de ce type de texte", voir B. Delforce, 1985) plutôt que comme preuves de leurincapacité... 
-Une même problématique de l'échec: à qui la faute ?La correction des copies crée chez les enseignants un certain sentiment d'inefficacité etd'échecdevant lequel ils se sentent démunis.Leurs hypothèses explicatives privilégient laresponsabilité desélèves:manque d'attention et de motivation pour écouter, insuffisance de la réflexion pour comprendre, absence de travail et d'intérêt pour retenir et appliquer... Ces hypothèses reposent, sur des représentations du savoir (simple transmission de connaissances) et du faire (simple application de celles-ci).

De leur côté, les élèves ont tôt fait, eux aussi, de renvoyer aux enseignants laresponsabilité de leurs mauvaises notes: l'enseignant "n'explique pas bien en classe", "on ne comprend pas ce qu'il veut", "il n'a pas luattentivement la copie"...

A y bien regarder, c'est la même question qui sous-tend ces deux réactions. Devantl'échec de ce qu'on a cherché à réussir, on ne se demande que :à qui la faute ?Par ailleurs, ces deux réactions reposent surun même horizon d'attente idéal l'absence d'erreurs comme situation normale et leur présence comme une "anomalie". Enfin, les hypothèses explicatives de l'échec témoignent, chez les uns et les autres, des mêmes représentations du savoir et du faire (attention, compréhension, clarté...).

6 Ici aussi, on peut efficacementdéplacer la problématiquesous-jacente : si on considère l'erreur transitoire comme un phénomène normal dans le processus d'appropriation et d'application du savoir (B. Delforce, 1982 b ; Y. Reuter, 1984), alors il n'est plus nécessaire de rechercher qui est coupable de ce qui n'est plus une "anomalie". Cette fois, la question centrale consiste à se demander comment le sujet en est arrivé defaçon logique etcohérente pour luiau résultat erroné qu'on a sous les yeux pour savoircomment améliorer les résultats (où et comment intervenir). A une problématique qui favorisel'explication de l'échecpar toutes sortesd'incapacitésoude manqueschez le sujet, on substitue une problématique centrée sur larésolutionde l'échecqui rend compte de son apparition par lesystème de connaissancespropre au sujet et les capacitésqu'il a déjà partiellementacquises.

57 -Une même méconnaissance: la nature de l'activité de l'autre.On l'a vu, les enseignants disposent, en réalité, de peu d'informations sur les conditions dans lesquelles sont réalisés les devoirs, sur les procédures de travail mises en oeuvre et sur les difficultés qui s'y font jour. De leur côté, les élèves sont loin de soupçonner que la correction des copies présente des difficultés et soit l'objet d'une réflexion chez les enseignants (voir, ici-même, Agnès Laurent). De même, au verdict des enseignants: "l'élève a "bâclé" son devoir" semble répondre, chez les élèves, un : "le professeur n'a pas bien lu ma copie".

Cesoupçon réciproquetémoigne, en fait, d'uneméconnaissancede l'activité de l'autre :qu'est-ce que lire et évaluer une copie ? inversement, qu'est-ce que produire un écrit scolaire?

Ainsi, les enseignants écriront: "Vous n'avez pas fait de plan",négligeant le fait que l'élève ait pu s'efforcer d'en faire un, sans réussir à le mettre en évidence pour que le lecteur ne s'y trompe pas. De même, ils écriront "maldit", "Penséeconfuse", "Contradiction", sans faire la part entre ce qui se pense, cequi cherche à s'exprimer et ce qui peut aisément se lire (voir les effets différents de : "ici, j’ai dû relire 3 fois avant de comprendre...", "j’ai hésité entre deux interprétations : soit... soit...", "à la lecture, cela paraît contradictoire, que voulais-tu dire ?", où le correcteur se situe résolument comme lecteur). De leur côté, les élèves accuseront: "vous n'avez pas compris ce que je voulais faire/dire", ne soupçonnant pas qu'un contenu ou un projet n'existe pas indépendamment de sa lecture. Bref,chacun perçoit l'activité de l'autre comme évidente et non-problématique. 
60 D'un côté, aussi, des élèves qui rédigent, régulièrement, des écrits, peu variés de surcroît, et qu'ils n'adressent jamais qu'à un seul lecteur/correcteur: on peut penser qu'ils accumulent ainsi une expérience concrète et intéressante des problèmes posés par l'écriture et la production des écrits scolaires, mais qu'ils ne peuvent vraiment en tirer parti faute de pouvoir, au moins, les verbaliser et les réfléchir.Que savons-nous de ces difficultés? que faisons-nous de cette expérience?

61 De l'autre côté, des enseignants qui ont rarement à rédiger des écrits pour un lecteur (J.F. Halté, A. Petitjean, 1980, p. 9) et qui, en tout cas, ne rédigent plus d'écrits scolaires, mais qui, à l'inverse, sont gros lecteurs de copies et qui pourraient avoir, de fait, accumulé une expérience des conditions de fonctionnement/dysfonctionnement des écrits, notamment, scolaires.Que tirons-nous de cette expérience? Qu'en savent les élèves?

Ici non plus,la situation n'a rien d'inéluctable: on peut aider de multiples manières à mieux percevoir les liens réciproques entre les opérations de lecture et d'écriture et à mieux conceptualiser les difficultés dont elles sont le lieu.

3.1.3) Conclusion :

63 En définitive, si le discours des élèves et celui des professeurs se présentent comme deux discours qui se maintiennent réciproquement en état d'équilibre, dans la mesure où ils partagent les mêmes problématiques implicites et les mêmes représentations, desdéplac ementsde celles-ci peuvent briser cette circularité et enclencher unenouvelle dynamique.

3.2) Comprendre le "point de vue" des élèves ?

On peut aussi se demander si ce discours des élèves est bien un discours mensonger et faux qui travestirait la réalitéou s'il n'est pas, au contraire, un discourscohérent, pertinentet adéquat à la réalité de l'évaluation,telle du moins que les élèves peuvent en percevoir le fonctionnement sur la base des représentations dont ils sont porteurs.

Cette dernière hypothèseexige qu'on éclaire le fonctionnement de l'évaluation non pas d'unpoint de vueillusoirementgénéralet prétendumentneutre,maisdu point de vue des élèves (J.P. Darré, 1978, chapitre 1 ; P. Bourdieu, 1980, p. 22).

En effet, la façon dont les individuss'approprient un phénomène de leur environnement, la nature et la signification,pour eux,de ce phénomène, ne leur sont pasdonnéspar les mots ou par les définitions qu'on leur en propose. Ils sont progressivementconstruitsà partir de l'ensemble des éléments de réalité - dont les mots et les définitions font partie - auxquels ils sont confrontés et à travers la façon dont ils les ressentent et les perçoivent.Les représentationsqu'on se construit de la réalité correspondent donc à unenécessité :rendre le réel intelligible pour savoir comment se comporter à son égard. Elles ne sont pas non plus untravestissementerroné ou volontairement habile de la réalité, mais une façon particulière de la connaître et de se l'approprier (Moscovici, 1976).

67 Par ailleurs, si la perception du réel et, indissolublement, le discours que nous tenons ainsi que les comportements que nous adoptons, dépendent du système de représentations que nous nous sommes construits, ils sont aussi le produit de laplacequ'on occupe et àpartir de laquelle on ne peut que percevoir la réalité d'une certaine façon. Là où on se trouveplacé,il y a des éléments que l'on perçoit bien, d'autres qu'on peut entrevoir ou deviner, d'autres, enfin, qui se trouvent cachés et dont on ignore même l'existence.

68 C'est pourquoi, si certains comportements ou propos des élèves paraissent proprement incompréhensibles si on les écoute à partir de ce qu'est l'évaluationdu point de vue de 
l'enseignant,il n'en va plus de même si on les rapporte aupoint de vue desélèves :ils résultent, alors,logiquement,des représentations de l'évaluation qu'ils ont $\mathrm{pu}$ construire. L'enseignant peut-il espérer faire évoluer les pratiques de l'évaluation chez les élèves (comportements, discours...), s'il necomprendpas les représentations qui les informent et les justifient à leurs yeux et si, donc, il ne peut modifier ses propres réactions d'hostilité ou de refus, à leur égard (perception du "bon" et du "mauvais" élève...).

3.2.1) Des représentations adéquates de l'évaluation.

-L'évaluation: un fonctionnement difficile à comprendreLa plupart des représentations de l'évaluation semblent empreintes de laréelle difficultédes élèves àcomprendre/construire ses règlesde fonctionnement. Beaucoup de propos ne sont que des variations sur un thème unique : "Je ne comprends pas bien pourquoi..." Les réactions témoigneraientd'abordde cette incompréhension avant même d'être des "excuses" ou des "ruses".

Et cetteincompréhensionne devrait pas, en définitive, nous surprendre tant. Ilne manque pas d'éléments dans le fonctionnementréelde l'évaluation qui peuvent la rendre "incompréhensible" du point de vue de l'élève. Lessujets proposésd'abord: au flou des consignes (voir, ici même, Y. Reuter) et à leurs exigences implicites voirecontradictoires (voir Y. Lefranc, 1985, chapitre 5 et ici même) s'ajoutent des conditions de communication artificielles et qui contreviennent à ses règles habituelles (voir J.F. Halté, 1981) et une indécision sur les finalités réelles de l'exercice (voirB. Delforce, 1985) sans parler d'une imposition subreptice des contenus (voir Y. Lefranc, et, ici même, Y. Reuter, note 3). La correctionensuite : c'est peu dire que les critères en restent flous et non systématiques; en tout cas, ils sont rarement explicitement affichés et ils restent "occultes" aux yeux des élèves. Ceux-ci ne peuvent, d'autre part, que très difficilement tirer parti de nos annotations et de nos corrigés pour comprendre "ce qu'il faudrait faire la prochaine fois" : nos annotations sontrarementexplicatives(voir J.F. Halté, 1984); elles n'expliquent jamais les erreurs en redéfinissant lesfinalités de l'exercice(voir B. Delforce, 1985); elles fonctionnent à partir denotions évaluativeset nonopératoires(voir B. Delforce, 1983) ; enfin, elles mettent plus l'accent sur lecas particulierque constituece devoirque sur une méthodologiepropre à cetype d'écrit spécifique(voir B. Delforce, 1985), rendant malaisé le transfert d'apprentissage.

71 Face à cette difficulté d'assigner à l'évaluation des principes de fonctionnement clairs, l'élève en est remis, dans le meilleur des cas, à tâtonner, à formuler des hypothèses et à chercher à les vérifier progressivement au fil des corrections. Les réactionsd'étonnement des élèves devant leur copie,l'échangede celles-ci entre voisins, lesquestionsposées, certainesphrasestypées...pourraient bien être, aussi, le signe de cetravail d'élaboration.

-Des représentations adéquates... mais restrictives.Dans cette perspective, certaines façons de se représenter l'évaluation, si elles sont restrictives à nos yeux, sont, du moins, tout à fait adéquates au fonctionnement réel de l'évaluation.

Ainsi,l'intérêt exclusif des élèves pour la note :c'est son aspect le plus aisément perceptible et le seul qui se prête bien pour eux à des comparaisons. C'est, en outre, le plus lourd de conséquences : dudevoir, l'institution ne retient qu'elle, la perpétue sur les "bulletins" et c'est elle qui déterminera le "destin-scolaire". D'ailleurs, les élèves se montrent proches, ainsi, des enseignants qui s'interrogent plus sur lanotation(Faut-il noter? ne pasnoter? Comment mieuxjugerles devoirs?) que sur lesannotations(comment les formuler pour qu'elles soient utiles et efficaces?). 

règlent leurs comportements,en dissociant mieux les deux fonctions de l'évaluation:l'une, formative, ne concerne directement que l'enseignant et l'élève: elle ne requiert pas absolument la note mais elle exige, par contre, une clarification des consignes et des finalités desexercices, un approfondissement des critères d'appréciation et de la formulation des annotations...; l'autre, sommative, exige surtout la note. Elle ne relève plus vraiment de la relation d'enseignement/apprentissage entre le professeur et l'élève, mais s'impose institutionnellementà l'un comme d l'autre(i.e., leur seul accord ne suffirait pas à la supprimer): l'enseignant n'en est donc pas le maitre "tout puissant". Appréciations et annotations n'y viennent quejustifier institutionnellementla note (pour l'administration et les parents autant que pour l'élève). base de cet aspect massivement mis en évidence, en classe, par les enseignants euxmêmes, comme la justification essentielle de l'évaluation, certains élèves pensent que l'exercice exige surtout uneréponse,voire, uneréponse unique(cf, par ex., l'exercice qui propose de "faireleplan du texte), et non laproduction d'un texted'une nature particulière : pour "expliquer une expression", on pense qu'il faut fournir uneréponsequi montre qu'on saitet non untextesusceptibled'expliquer/faire comprendrecette expression à quelqu'un. De même, les élèves ne comprennent plus alors que leur réponse, quicontient plusieurs éléments justes, ne reçoive pas une meilleure note, ou que deux plans qui sont, l'un et l'autre, très différents de "celui qu'il fallait trouver" aient des notes si différentes, ni, a fortiori, qu'une réponse pourtant juste (la définition du mot réécrite à partir du dictionnaire) n'obtienne pas une note excellente... Sans parler de l'exigence toujours implicite d'une application "personnelle" et non "mécanique" du savoir transmis ${ }^{4}$ (voir Y. Lefranc, 1985 et ici même). exercices et des modalités de leur appréciation peutaffiner les représentations et modifier, en conséquence, les comportements.

-Des représentations très restrictives... mais toujours adéquates.Mais il se pourrait aussi que, faute de percevoir desrégularitésutilesou de comprendrecommentils pourraientaméliorer leurs propres écrits, certains élèves en arrivent,progressivement,à penser que ce sont seulement la"côte"dont on bénéficie auprès du professeur ou"les facilités"qu'ont certains, qui expliquent les résultats. mauvaises notes échoient souvent aux mêmes. D'autres éléments du fonctionnement de l'évaluation peuvent ensuite venir "confirmer" ces représentations.

79 Ainsi, le sempiternel "Votre devoir n'a pas deplan", quand on sait pertinemment en avoir fait un, conduit à penser que décidément" on nous en veut ".Vont progressivement dans le même sens: l'impossibilité d'expliquer les différences entre les notes ou l'impression qu'on a été "sanctionné pour sesidées". En effet, puisque le "contenu" est, à leurs yeux, l'attente essentielle de l'enseignant (voir la formulation des sujets), ce sont bien les idées émises qui doivent expliquer l'écart entre les notes, même - et peut-être surtout - si l'enseignant n'aborde pas cet aspect dans les annotations ("c'est tellement inattendu, que ça doit bien cacher quelque chose d'inavouable").

80 L'impression qu'il faut"avoir des facilités"peut, elle aussi, trouver des confirmations dans le fonctionnement de l'évaluation comme dans le discours, au moins tacite, des enseignants. 
N'entendent-ils pas, au moins à demi-mot, que l'écriture est affaire d'imagination ou de logique, voire d'inspiration ou de génie? Ne sont-ils pas sensibles au caractère brillant et original du corrigé proposé... qu'ils se sentent bien incapables de produire ? Le professeur ne leur dit-il pas que "c'était pourtant facile"... quand ils ont l'impression, eux, qu'il n'en était rien? Bref, tout les porte à croire, comme, en définitive, les enseignants, que certains ont des "difficultés" et que d'autres ont des "facilités" ; car si le mot "don" recule, il n'est pas sûr que l'idée n'en soit pas reprise dans le terme "capacité" (voir G. Langouet, 1984, p. 29 ; B. Delforce, 1985, pp. 36-37).

81 Enfin, certains élèves semblent ne pas parvenir du tout à établir des régularités. Ils assimilent alors l'évaluation à uneloterie, un jeu de hasard. Voilà qui peut surprendre, mais qui n'est jamais tout à fait absent du discours des enseignants (cf les plaisanteries sur "l'escalier" du correcteur). Et comment nommer un jeu dont on neperçoitpas lesrègles,ni lesrégularités,et dont on ne parvient jamais àprédireles résultats? Les élèves, comme d'autres, attribuent au hasard ce dont ils ne peuvent expliquer l'apparition. D'ailleurs, sommes-nous sûrs quetousles élèves perçoivent même les régularités, massivement évidentes à nos yeux, qui relient les exercices faits en classe au devoir proposé ou un devoir à ceux qui l'ont précédé (voir, par ex., L. Demailly, 1979, p. 33 ; V. Isambert-Jamati et M.F. Grospiron, 1984, p. 95).

D'où, sans doute,la fréquence du thèmechance/malchancedans le discours des élèves et cette réaction derésignationdevant les coups du sort. On comprend mieux aussi pourquoi certains élèves s'ingénient à percevoir à tout prix desrégularités.'là où nous ne voyons que phénomènes aléatoireset sans signification ("Il aime les plans en combien de parties ?" "Quelles citations il apprécie ?"...).

* Conclusion :

En définitive, certains élèves semblent progressivement conduits à se construire des représentations de l'évaluation fondées surl'irrégularitétotale ou sur desrégularités,parfois trèsrestrictives,mais toujours adéquates au fonctionnement réel et qui ne sont "simplistes" qu'à notrepoint de vue d'enseignants.Les plus élaborées les détournent de la recherche de régularités plus pertinentes et plus productrices; les autres ("avoir la côte", "avoir des facilités", "une loterie") les "démobilisent" (à quoi bon faire des efforts si "on n'y peut rien" ?), amplifiant ainsi leur situation d'échec.

85 Sans doute ces façons de désigner l'évaluation résultent-elles parfois, nous le verrons, d'une "ruse". Mais peut-on affirmer que ce soit le cas cheztous? Que ce soit le cas,d'emblée, chez ceux qui, petità petit, en tirent ce parti ?

3.2.2) Des représentations stratégiquement pertinentes.

86 Tout discours engage l'image de soi du locuteur, ou, au moins, l'image de soi comme locuteur susceptible de dire des choses intéressantes et de les direscomme il convient. Bref, l'enjeu y est toujours aussi d'être un locuteur digne d'être écouté et reconnu (voir, par ex., P. Bourdieu, 1982, pp. 59-60 ; ou encore F. Flahault, 1978, Y. Lefranc, 1985).

Cet enjeu de tout discours nous paraît particulièrement net dans la correction des copies telle qu'elle fonctionne. D'abord, les sujets proposés invitent le locuteur à yinvestir de soi, qu'il s'agisse de raconter (voir J.F. Halté, 1981) ou de disserter (voir B Delforce, 1985). D'autre part, les corrections répétées vont conduire a des "appréciations" trimestrielles portant plus surl'individuque sur sesperformances.Pour cette double raison, tout jugement/ appréciation porté sur le devoir se trouvera donc êtreaussiun jugement porté sur l'individu et reçu comme tel. 
Devant l'importance de l'enjeu, il devient tout à fait pertinent de chercher systématiquement à faire reconnaître sa valeur (scolaire et de sujet). Et faute de pouvoir donner - ou d'espérer recevoir - de soi une image positive, on peut s'efforcer, en échange, de seprémunir contre toute atteinte disqualifiante,au moins à ses propres yeux, ainsi qu'à ceux du groupe de pairs.Plusieursstratégies pertinentess'offrent alors, qui pourraient bien rendre plus "compréhensibles" certains propos ou certains comportements chez les élèves.

On peut, d'abord, chercher àdonner au verdict une significationsystématiquement qualifiante. Cela pourrait bien être la fonction essentielle des nombreusesprédictions négativesà l'issue des devoirs et expliquer aussi certaines réactionsd'étonnementau retour des devoirs. En effet, si je prédis que "j'aurai une mauvaise note", deux issues sont possibles: ou bien j'obtiens, de fait, une bonne note et je bénéficie ainsi d'une image positive ("j'ai réussi") ; ou bien, j'obtiens effectivement une mauvaise note, mais je suis valorisé par la justesse de ma prédiction ("je ne m'étais pas trompé"). Dans les deux cas, je me protège partiellement contre la disqualification, alors que la prédiction inverse n'offre pas de bénéfice supplémentaire sielle se vérifie, mais est doublement disqualifiante si elle se révèle fausse.

On peut, d'autre part, se prémunir d'un verdict négatif enle vidant de sa signification.Ainsi, si la correction des copies est assimilée à uneloterie,je peux espérer tirer des "bénéfices compensatoires" de cette façon de désigner le jeu : outre qu'il est peu coûteux d'avouer qu'on n'a pas de chance ${ }^{(5)}$, on peut même espérer ainsi s'attirer la sympathie des autres, surtout s'il apparaît que la malchance vous frappe injustement ("J'avais pourtant travaillé")6. De même, si l'on désigne le verdict comme résultant de la"côte"dont on bénéficie : outre qu'on n'y peut rien, on peut même parfois en tirer "gloire" auprès du groupe de pairs ou avoir de bonnes raisons de se "retirer du jeu" ou de s'y montrer indifférentsans avoir l'air de "démissionner" (Il peut être disqualifiant, pour certains, de passer pour un "dégonflé").

Une autre façon de se protéger consisterait àinverser subrepticementle sens du jeu.Certains propos ("le professeur n'explique pas bien", "Personne n'a réussi"...) remettent au premier plan l'autre fonction du professeur : non plus corriger, mais enseigner, c'est-àdire,faire/réussirles apprentissages. Dans cettenouvelle définition du jeu,l'élève récupère le pouvoir de verdict et son propre échec peut disqualifier l'enseignant (qui pourra toujours s'y montrer, à son tour, indifférent!). De même, les "J'ai toujours été nul" peuvent-ils constituer undéfià relever ("Saurez-vous y changer quelque chose?!)... quel'enseignant ne pourra gagner qu'à coup de notes meilleures ! La - rare -bravade("c'est moi qui ai eu la plus mauvaise note !) pourrait aussi signaler l'existence d'une espèce decontrat taciteentre certains membres du groupe : celui d'une résistance au moins passive au jeu, qui invite à ne pas collaborer "exagérément". On pourrait alors tirer "gloire" d'avoir respecté au plus haut point ce contrat, la bravade ne serait ainsi qu'une forme extrême d'un phénomène dontl'indifférence ostensiblemarqueraitla forme moyenne ("si vous croyez me toucher punir/récompenser - avec vos notes!"). Certaines réactionsd'étonnementdevant des bonnes notes pourraient aussi signifier publiquement l'allégeance à ce contrat: "je n'ai rien fait pour", "je n'y suis pourrien".

Enfin, une dernière façon de se protéger contre la disqualification consisterait à disqualifier le jeu lui-même.Quelle valeur peut avoir le verdict négatif d'un jeu qu'on perçoit comme "faussé" ou qu'on trouve "sans intérêt" ? Certains éléments dudiscours critique 
contre l'évaluation ("c'est injuste", "on est saqué"...) ou contre l'école ("c'est idiot", "ça ne sert à rien"...) pourraient aussi remplir cette fonction.

Pour prolonger les perspectives évoquées ici, on peut s'interroger surl'efficacitédesdeux appréciationsqui servent de base à la rédaction des bulletins et qui conjuguent les deux critères centraux des "capacités intellectuelles" et de "l'effort" :"bonnevolonté mais faible" et"Élève intelligent qui ne fait rien".On peut craindre,en effet, qu'elles ne soient également dissuasivespour certains élèves. La première risque fort de produire ledécouragement(à quoi bon, si l'onestfaible...). La seconde pourrait bien avoir le même effet: si l'élève travaille et qu'il réussit, tout va bien (il n'a rien gagné d'essentiel, mais il n'a rien perdu); par contre, il peut craindre de compromettre son image positive s'il apparaît qu'il travaille sans que ses résultats s'améliorentnotablement.Il vaut mieux alors bénéficier a priori d'uncréditd'intelligence que d'avoir à en manifester lapreuve.

En définitive, ces diverses façons de "refuser" le jeu font apparaître en filigrane tout ce à quoi il fautcroireet les valeurs auxquelles il fautadhérerpour accepter de le jouerpleinement, d'ensupporter le verdict et, en conséquence, de s'investir dans les apprentissages qui lui sont liés. Ce serait l'objet d'un autre travail'?

3.2.3. Des représentations socialement légitimes.

Le discours et les réactions des élèves manifestent de telles régularités qu'ils semblent indépendants des élèves qui le portent, des enseignants à qui ils s'adressent ou des situations scolaires spécifiques où ils s'énoncent. En ce sens, cediscours (verbal/non verbal) seraitindépendant des individusqui l'expriment etsignificatifsurtoutdes caractéristiques structurellesdes situations qui en sont l'occasion. Il se présenterait comme un "stock" d'éléments différenciés et disponibles selonles besoins.

C'est l'hypothèse que nous avons privilégiée jusque là en rapportant systématiquement ce discours, d'une part, àceluides professeursauquel il répond et avec lequel il forme système, d'autre part, àl'évaluation telle qu'elle fonctionne dupoint devue des élèves.

Pourtant, des remarques, non systématisées mais convergentes, nous incitent à rapprocher aussi ce discours des individus qui le portent. Les représentations de l'évaluation ne feraient que reproduire à son propos certains traits devisions du monde plus globales. On s'en tiendra ici à quelques remarques rapides en ce sens.

Précisons d'abord que l'évaluation telle qu'elle fonctionneconcrètement -et quelles qu'en soient les modalités précises - est toujours implicitement porteuse devaleurs et de significations sous-jacentes: y adhérerpleinementsuppose qu'on partage le système de représentations et de valeurs qu'elle exprime. D'autre part, la construction de représentations diversifiées de l'évaluation est autant le fait des élèves que des enseignants : en ce sens, la mise à distance, ou "l'hostilité" à l'égard de certains éléments du discours des élèves, ne ferait que traduire une distance culturelle et sociale à d'autres visions du monde.

-Fatalisme ou changement ?Lareprésentation de l'évaluation commeloterieet les attitudes fatalistes et résignées qu'elle engendre rappellent les observations des sociologues à propos de certaines fractions de la classe ouvrière. C'est que, pour adopter une attitude plus dynamique, il faut avoir de bonnes raisons de croire, d'une part, que ce qui arrive n'est ni l'effet d'un quelconquehasardni celui d'une incontournable"nature",d'autre part, qu'on peut changer quelque chose à des situations qui n'ont rien d"éternel". Viendraientsans difficulté s'intégrer à ces visions du monde statiques les représentations 
des capacités comme "dons naturels". ("Il y a des gagnants et des perdants", "Il faut bien des intellectuels et des manuels"...).

-Compétition ou solidarité? Les valeurs decompétitionet deréussite personnelleque porte l'évaluation peuvent être à la convenance de groupes sociaux où laréalisation de soi et l'individualismede la réussitesont des valeurs, en quelque sorterequises,par leur situation concrète. D'autres groupes ont, au contraire, été en situation de développer des valeurs decoopérationet de solidarité: jouer pleinement le jeu de l'évaluation dans ses formes dominantes, cela pourrait bien signifier " vouloir être au-dessus des autres" et être ressenti commetrahison des valeurs de "promotion collective" (cf. la fréquence du thème de la trahison chez les autodidactes). De même, si certains peuvent croire que leur seule voie de promotion sociale passe par la réussite scolaire (fils de fonctionnaires, même de catégorie D), d'autres savent pouvoir, si nécessaire, s'en passer quand d'autres n'ont vraiment aucune raison objective d'y croire. Si les uns peuvent valoriser l'évaluation et les "indispensables" valeurs de mérite personnel (travail, effort, compétence...) et de justice qu'elle accomplit, les autres peuvent avoir quelques raisons d'y être moins "sensibles".

-Modestie ou ostension ?L'évaluation exige fortement non seulement des compétences, mais aussi unemise en scènede celles-ci : il importe avant toutde les rendrevisiblesdans unemise en valeur de soi,qui permet même de paraître plus compétent qu'on est (voir P. Perrenoud, 1979, pp.39-40). Certains groupes sociaux qui se sont "habitués" à valoriser des comportements demodestiepeuvent très bien ne pas y retrouver leur compte (cf. "vouloir péter plus haut qu'on a le cul", "se prendre pour quelqu'un"...).

-L'école et l'usine: Certains élèves pourraient bien nouer aussi à la situation scolaire et cristalliser sur l'évaluation desmodesde relationvalorisés d l'usine.ll semble, en tout cas, intéressant de chercher dans cette voie, notamment autour des rapports autravail,à la productionet à lahiérarchie.A l'usine aussi on revendique le salaire comme une juste rétribution du temps de travail fourni - indépendamment de la nature et de la qualité de la production-; là aussi, il peut être gravement disqualifiant de trop collaborer et d'accélérer les cadences : il est de bonne guerre de rester juste à la limite inférieure des cadences proposées ; làaussi, il est patent qu'on peut être "saqué" pour ses idées ; là aussi, on valorise celui qui sait "ouvrir sa grande gueule" (voir P. Bourdieu, 1982, p. 93) et on refuse la "docilité" à l'égard des "petits chefs". Bref, si l'école prépare à des comportements adaptés à l'usine, les élèves s'y prépareraient, quant à eux, à y résister.

\section{BIBLIOGRAPHIE}

Bourdieu, P., (1980),Questions de sociologie,Paris, Edition de Minuit.

Bourdieu, P., (1982), Ce que parler veut dire,Paris, Fayard.

Charolles, M., (1980), "Les formes directes et indirectes de l'argumentation",Pratiques, n $^{\circ} 28$, octobre 1980.

Darre, J.P., (1978),Liberté et efficacité dans les groupes de travail,Paris, les Editions Ouvrières. 
Delforce, B., (1982 a), "A quoi ça peut bien me servir de travailler sur des textespuisque je suis venu pour apprendre à mieux m'exprimer?! Activités de lecture et activités d'écriture en techniques d'expression".Expressions, ${ }^{\circ}$ 36, octobre 1982 (Bull. Pédag. des IUT, n 79).

Delforce, B., (1982 b), " Les difficultés langagières. Pour une autre hypothèse explicative : le rôle des représentations".Bulletindu CERTE,n 1, décembre 1982.

Delforce, B., (1983), "Pour une didactique des écrits pratiques : la note de synthèse",Bulletin du CERTE,n 3, juin 1983.

Delforce, B., (1985), "Approches didactiques de la production d'un écrit "fonctionnel" : les difficultés de la dissertation"Pratiques,nº 48, décembre 1985.

Demailly, L., (1979), "Diversités des pratiques pédagogiques"Pratiques,nº spécial, "Colloque de Cerisy".

Flahault, F.,(1978),La parole intermédiaire,Paris, Seuil.

Halté, J.F., Petitjean, A., (1980), "Écrits, Écriture et Société"Pratiques,n² 26, mars 1980.

Halté, J.F., (1981), "Pour changer l'écrire",Pratiques,n² 29, mars 1981.

Halté, J.F., (1982), "Apprendre autrement à l'école"Pratiques,nº 36, décembre 1982.

Halté, J.F., (1984), "L’annotation des copies",Pratiques,nº 44, décembre 1984.

Isambert-Jamati, V., Grospiron, M.F., (1984), "Types de pédagogies du français et différenciation sociale des résultats. L'exemple du "travail autonome" au 2ème cycle long".Études de LinguistiqueAppliquée, $\mathrm{n}^{\circ}$ 54, avril-juin 1984.

Langollet, G., (1984), "L’enseignement du français dans les collèges : élèves et enseignants". Étudesde Linguistique Appliquée,nº 54, Avril-juin 1984.

Lefranc, Y., (1985), "Un exercice d'argumentation en situation scolaire algérienne.". Thèse de 3e cycle - E.H.E.S.S. Paris.

Moscovici, S., (1976),La Psychanalyse, son image et son public,Paris, P.U.F., (1ère édition 1961).

Perrenoud, P., (1979), "Des différences culturelles aux inégalités scolaires : l'évaluation et la norme dans un enseignement indifférencié", inL'Évaluationformative dans un enseignement différencié,L. Allai (éd.), Berne, P. Lang.

Reuter, Y., (1984), "Pour une autre pratique de Terreur".Pratiques,nº 44, décembre 1984.

\section{NOTES}

1. Sur la reconstitution des difficultés de ce travail pour l'élève, voir J.F. Halté, 1984.

2. Toutes les références au discours des enseignants renvoient à B. Delforce, ici-même.

3. Pour une hypothèse qui privilégie davantage l'antagonisme, voir Y. Lefranc, 1985, et ici-même.

4. En outre, sur l'exigence d'une application non mécaniquede ce qu'a transmis l'enseignant, voir

Y. Lefranc, 1985 et ici-même.

5. Dans le monde scolaire et dans celui du travail social en tout cas. 11 semble, par contre, en aller différemment dans celui des cadres d'entreprise.

6. Cf. l'idéologie du mérite: quand le malheur vous frappe, il vaut mieuxne rien avoir à se reprocher.

7. Dont le modèle pourrait nous être fourni par l'article de M. Charolles 1980. 


\section{RÉSUMÉS}

Lanote donnée à une production écrite est la première chose qu'attend et que perçoit l'élève. La réaction verbale des élèves à celle-ci est un phénomène récurrent selon l'auteur. Il analyse ici ce discours facilement accessible, par l'observation passive d'une classe, pour tenter d'en comprendre les ressorts. Il s'agit de savoir si les enseignants peuvent le prendre en compte pour améliorer leur pratique pédagogique ou si ce discours n'est que factice et seulement spontané.

INDEX

Mots-clés : enseignement, élève, évaluation, analyse de discours

Keywords : education, pupil, rating, discourse analysis

\section{AUTEUR}

\section{BERNARD DELFORCE}

Bernard Delforce,Université de Lille III 